\title{
Flow-volume curve in the diagnosis and follow-up of intrathoracic airway obstruction
}

\author{
Özlem Cavkaytar ${ }^{1}$, Ayşe Büyükçam² ${ }^{2}$ Özlem Tekşam² ${ }^{2}$, Deniz Doğru-Ersöz ${ }^{3}$, Zuhal Akçören ${ }^{4}$, \\ Umut Akyol ${ }^{5}$, Ayfer Tuncer ${ }^{1}$, Cansin Saçkesen ${ }^{1,6}$ \\ Divisions of ${ }^{1}$ Pediatric Allergy, ${ }^{2}$ Pediatric Emergency, ${ }^{3}$ Pediatric Pulmonology, and ${ }^{4}$ Pediatric Pathology, Department \\ of Pediatrics, ${ }^{5}$ Department of Otorhinolaryngology-Head and Neck Surgery, Hacettepe University Faculty of Medicine, \\ Ankara; ${ }^{6}$ Division of Pediatric Allergy Department of Pediatrics, Koc University School of Medicine, Istanbul, Turkey. \\ E-mail: csackesen@ku.edu.tr
}

Received: 24th May 2016, Revised: 10th August 2017, Accepted: 16th August 2017

SUMMARY: Cavkaytar Ö, Büyükçam A, Tekşam Ö, Doğru-Ersöz D, Akçören Z, Akyol U, Tuncer A, Saçkesen C. Flow-volume curve in the diagnosis and follow - up of intrathoracic airway obstruction. Turk J Pediatr 2017; 59: 594-597.

Spirometry is an easy method to measure lung function and to show pathophysiology. It assists not only to determine the severity of bronchial obstruction in asthma but also to differentiate the characteristics of the intrathoracic diseases narrowing the central airways. Different types of benign and malignant tumors of the trachea may cause emergence of symptoms of airway obstruction. Herein a patient who had been initially diagnosed with asthma but later on shown to have intratracheal myofibroblastic tumor is presented. The importance of flow-volume curve in both initial diagnosis of the mass and in the detection of recurrence is discussed.

Key words: airway obstruction, asthma, flow-volume curve, inflammatory myofibroblastic tumor, intratracheal tumor.

Spirometry is a noninvasive and practical method to measure lung function, which leads to examine both inhalation and exhalation curves of the patient with symptoms of cough or shortness of breath. It assists not only to determine the severity of bronchial obstruction but also to differentiate the characteristics of the intrathoracic diseases narrowing the central airways. ${ }^{1,2}$ Spirometry is widely used in the diagnosis of asthma showing lower airway obstruction in that case. In lower airway obstruction the drop of flow is maximum at the beginning of expiration resulting in a concavity on flow-volume curve ${ }^{3}$. Spirometry is also used in detection and differentiation of upper and central airway obstruction. ${ }^{4}$

Primary tracheal tumors constitute rare group of disorders with a relative incidence to lung tumors as $1: 180 .{ }^{5}$ Different types of benign and malignant tumors of the trachea may cause emergence of symptoms of airway obstruction. The examples of these asthma mimickers reported previously are schwannoma, neurilemmoma, mucoepidermoid carcinoma, hamartoma. ${ }^{6}$ Besides these tumors, inflammatory myofibroblastic tumor (IMT) may also originate from trachea and has an intermediate degree of malignant potential with unknown etiology ${ }^{7}$ and a rarer incidence ranging from 0.04 to $0.7 \%$ of all lung masses. ${ }^{8}$

Here a patient who was referred with an initial diagnosis of asthma with a compression in both inspiratory and expiratory loops on flow-volume curve and diagnosed later with an IMT is reported and the importance of flowvolume curve in the follow-up of the patient is pointed out.

\section{Case Report}

A ten-year-old girl was admitted to Hacettepe University Division of Pediatric Allergy with the predominant complaints of chest tightness and wheezing, which were aggravating especially at nighttime and had been going on for one month. During this time she had had several admissions because of cough and dyspnea and had been hospitalized with a diagnosis of asthma. Since she was unresponsive to the 
treatment of oral corticosteroid, salbutamol and montelukast she was referred to our hospital. On physical examination she had tachypnea, stridor and suprasternal retraction and particularly the expiration phase of respiration was not heard on auscultation. Shortness of breath was aggravating suddenly and treatment with nebulized salbutamol, ipratroprium bromide and oral prednisolone were partially successful. Pulmonary X-ray was normal and the spirometric measurements were FVC 77\%, FEV1 59\%, FEV1/FVC 68\%, PEF $38 \%$ of the predicted values. The flowvolume loop demonstrated a plateau in both expiratory and inspiratory phase suggesting a fixed intrathoracic obstruction (Fig. 1A) rather than bronchoconstriction seen in asthma. The laryngotracheal endoscopic examination showed a polipoid tumor, three $\mathrm{cm}$ in diameter, originating from posterior wall of the distal one third of trachea, which obstructed nearly the entire lumen (Fig. 1B.). The mass was completely excised with clear surgical margins and there were no other pathologic findings in the bronchi. The pathologic examination of the mass determined IMT. The symptoms disappeared completely and the flow-volume curve returned to normal immediately after the excision (Fig. 2A). After six months a slight compression was observed again on flow-volume curve without any symptoms (Fig. 2B.). The tomography revealed a new polypoid mass, $10 \times 13 \mathrm{~mm}$ filling half of the tracheal lumen originating from the same location as the previous one without any other parenchymal or peripheral bronchial mass (Fig. 3). The mass was re-excised completely with the cauterization of the tumor origin on the posterior wall of trachea. The patient is followed-up with short intervals till then without any further recurrence. Informed consent was received from the patient and her parents.

\section{Discussion}

In this case report a patient with an initial diagnosis of asthma is presented without recovery despite appropriate treatment. Common symptoms of asthma like recurrent wheezing, cough and shortness of breath were also seen in this patient. But her response to treatment for asthma was partial. She had compressions on both inspiratory and expiratory loops of flow-volume curve suggesting a fixed upper central airway obstruction that limits airflow in both directions, which prompted the necessity for a direct laryngoscopy and bronchoscopy. Bronchoscopy revealed a tumor in intrathoracic part of trachea above the carina filling nearly the entire lumen. Uchida et al. ${ }^{9}$ reported another example of fixed airway obstruction caused by congenital tracheal stenosis located at thoracic inlet which was previously misdiagnosed with asthma as in our patient ${ }^{9}$. In our patient the changes on the flow-volume curve including inspiratory and expiratory phases helped us to differentiate the location of the mass in the central airways. Besides this, at the time of recurrence the mass was filling half of the
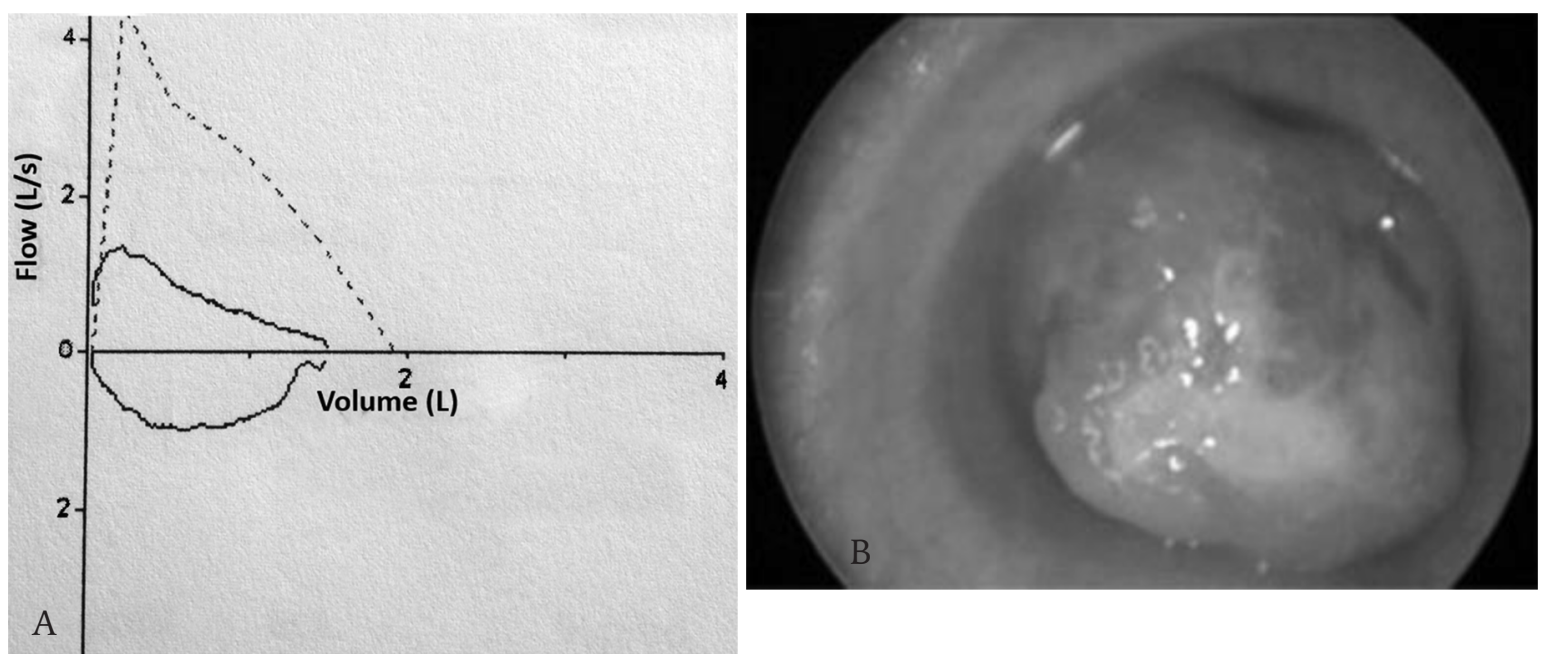

Fig. 1A. Flow-volume curve of the patient at the time of initial admission. B. The bronchoscopic image of the intratracheal mass. 

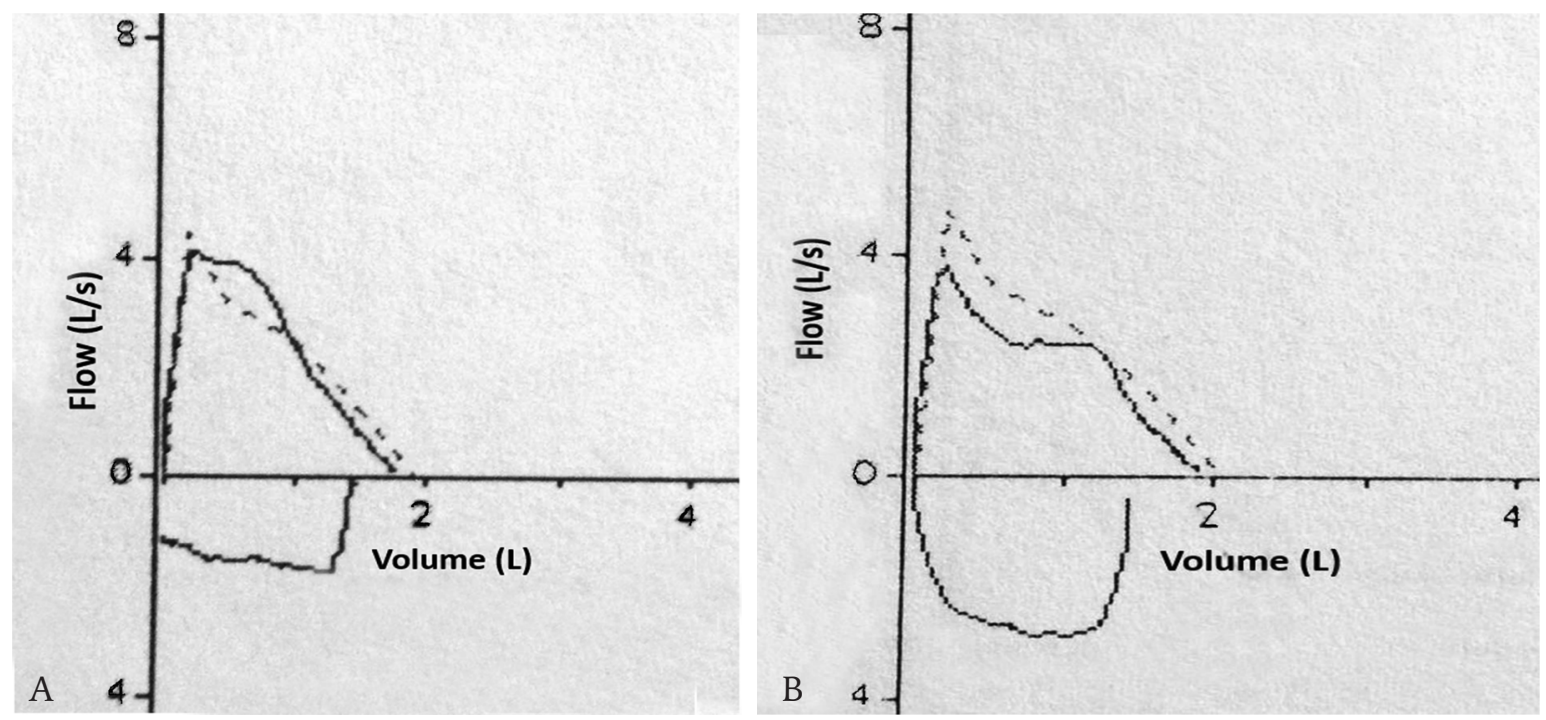

Fig. 2A. Flow-volume curve of the patient after the complete excision of the intratracheal mass. B. Flow-volume curve of the patients at the time of recurrence.

tracheal lumen and it was surprising that the compression was observed only in the expiratory phase of the flow-volume curve showing that the small size of the tumor permitted the airflow during inspiration but not on expiration. Our report pointed out for the first time the significance of flow-volume curve on spirometry in the follow-up of the patient even in the asymptomatic period indicating that the use of spirometry was reasonable and target driven in the follow-up as a screening method.

In children, IMT is seen primarily in the lung constituting $20 \%$ of all intrapulmonary tumors, however tracheal IMTs constitute a very small portion $(2.7 \%)$ of all respiratory tract associated IMTs $^{10}$. Although IMT was originally considered to be benign in origin, it has recently been classified as a low-grade neoplasm and the lesions filling the tracheal lumen may cause dyspnea and mimic asthma exacerbation. It is also named as inflammatory pseudotumor, xanthomatous pseudotumor, fibrous xanthoma, xanthogranuloma orhistiocytoma. ${ }^{10}$ Intratracheal IMT was reported in a total of 14 patients, nine of whom were in pediatric age group ${ }^{10}$ and then in 2008 five new pediatric cases ${ }^{11}$ were reported. The recurrence of tracheal IMT was also seen in a 2-year-old child who was symptomatic 3 months after excision of the initial lesion. ${ }^{12}$ It is important to note that in our case the recurrence was noticed due to the abnormal curve (Fig. 2B) on spirometry six months after the initial excision although the patient was asymptomatic.

Flow-volume curve on spirometry also aids in differentiating not only variable and fixed intrathoracic airflow obstructions but also extrathoracic ones. ${ }^{13}$ Spirometry is recommended in the follow-up of asthmatic children older than four years of age. ${ }^{3}$ It is useful in case the spirometric measurements are reproducible and consistent with each other at sequential maneuvers. Hopefully our patient was successful in producing objective loops in both expiratory and inspiratory phases of respiration and her capability made the spirometry an easy and practical diagnostic tool.

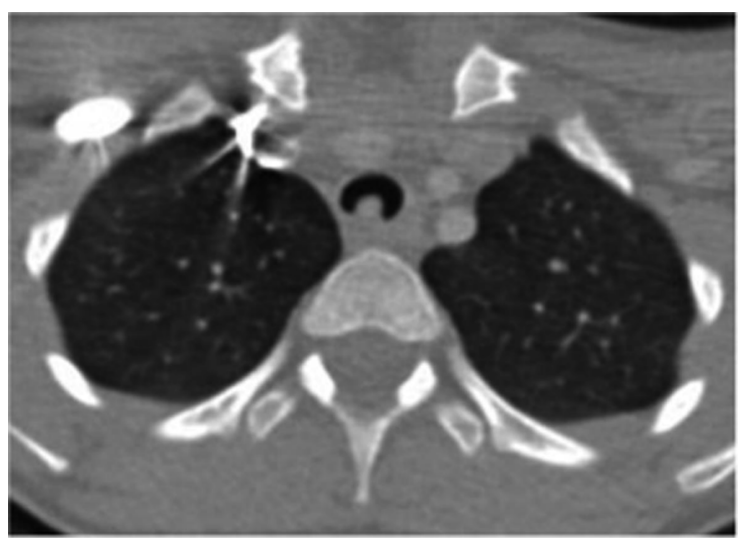

Fig. 3. Computer tomography scan of the recurrent intratracheal mass. 
In conclusion, the lesions filling the tracheal lumen may cause dyspnea and mimic asthma exacerbation. The characteristic flow-volume curve and unresponsiveness to asthma treatment are important clues to make differential diagnosis of airway obstruction and to guide for more invasive diagnostic methods. Moreover the flow-volume curve on spirometry is very useful in the follow-up to detect the recurrence of the mass even in a period without any symptoms.

\section{REFERENCES}

1. Hayes D, Kraman SS. The physiologic basis of spirometry. Respir Care 2009; 54: 1717-1726.

2. Parker MJ. Interpreting spirometry: the basics. Otolaryngol Clin North Am 2014; 47: 39-53.

3. From the Global Strategy for Asthma Management and Prevention. (updated 2015). Available at: http://www. ginasthma.org/. (Accessed Jan 12, 2016.)

4. Sterner JB, Morris MJ, Sill JM, Hayes JA. Inspiratory flow-volume curve evaluation for detecting upper airway disease. Respir Care 2009; 54: 461-466.

5. Houston HE, Payne WS, Harrison EG Jr, Holsen AM. Primary cancers of the trachea. Arch Surg 1969; 99: 132-140.
6. Roby BB, Drehner D, Sidman JD. Pediatric tracheal and endobronchial tumors: an institutional experience. Arch Otolaryngol Head Neck Surg 2011; 137: 925-929.

7. Mehta B, Mascarenhas L, Zhou S, Wang L, Venkatraman R. Inflammatory myofibroblastic tumors in childhood. Pediatr Hematol Oncol 2013; 30: 640-645

8. Hussain SF, Salahuddin N, Khan A, Memon SS, Fatimi $\mathrm{SH}$, Ahmed R. The insidious onset of dyspnea and right lung collapse in a 35-year-old man. Chest 2005; 127: 1844-1847.

9. Uchida DA, Morgan-Wallace V, Richards K, Seidelman J, Muntz HR. Congenital tracheal stenosis masquerading as asthma in an adolescent: The value of spirometry. Clin Pediatr 2009; 48: 432-434.

10. Bumber Z, Jurlina M, Manojlovic S, Jakic-Razumovic J. Inflammatory pseudotumor of the trachea. J Pediatr Surg 2001; 36: 631-634

11. Venizelos I, Papathomas T, Anagnostou E, Tsanakas J, Kirvassilis F, Kontzoglou G. Pediatric inflammatory myofibroblastic tumor of the trachea: A case report and review of the literature. Pediatr Pulmonol 2008; 43: 831-835.

12. Boloursaz MR, Khalilzadeh S, Dezfoli AA, et al. Inflammatory myofibroblastic tumor of the trachea. Pediatr Surg Int 2011; 27: 895-897.

13. Pellegrino R, Viegi G, Brusasco V, et al. Interpretative strategies for lung function tests. Eur Respir J 2005; 26: $948-968$. 